\title{
A Demonstration of Adaptive Weather-Surveillance Capabilities on the National Weather Radar Testbed Phased-Array Radar
}

\author{
Sebastián Torres, Ric Adams, Christopher Curtis, Eddie Forren, Douglas Forsyth, \\ Igor Ivić, David Priegnitz, John Thompson, and David Warde. \\ Cooperative Institute for Mesoscale Meteorological Studies, The University of Oklahoma \\ and NOAA/OAR National Severe Storms Laboratory, Norman, OK, USA.
}

\begin{abstract}
This paper describes the latest adaptive scanning capabilities of the National Weather Radar Testbed PhasedArray Radar located in Norman, OK. Focused observations, tailored observations, and the required scheduling algorithms are introduced, and their performance is illustrated with real-data examples. It is demonstrated that adaptive scanning for weather radars has the potential to reduce revisit times and to provide meteorological data that can aid in the forecaster's warningdecision process.
\end{abstract}

Keywords-weather radar; weather surveillance; adaptive scanning; phased array radar; NWRT PAR

\section{INTRODUCTION}

The U.S. government operates seven radar networks providing weather and aircraft surveillance for public weather services, air traffic control, and homeland defense. A nextgeneration, multifunction phased array radar (MPAR) concept has been proposed that could provide enhanced weather and aircraft surveillance services with potentially lower life-cycle costs than multiple single-function radar networks. As critical technology costs decrease, MPAR radars could prove to be a cost-effective alternative to current surveillance radars [1].

The National Weather Radar Testbed Phased-Array Radar (NWRT PAR) is an S-band phased-array radar located in Norman, Oklahoma that was established to demonstrate the MPAR concept. Since its inception, scientists and engineers at the National Severe Storms Laboratory (NSSL) have been improving the quality of data produced by this system and, more importantly, demonstrating new capabilities for weather observations. Unlike conventional radars, which are constrained by inertial limitations of mechanical scanning, the NWRT PAR can exploit electronic beam steering to focus and tailor weather observations on areas of interest without having to collect data contiguously.

In a nutshell, the NWRT PAR exploits a passive, 4352element phased-array antenna to provide stationary, twodimensional electronic scanning within a given $90^{\circ}$ azimuthal sector. The antenna is mounted on a pedestal so that the best overall orientation can be (manually or automatically) selected

Funding was provided by NOAA/Office of Oceanic and Atmospheric Research under NOAA-University of Oklahoma Cooperative Agreement \#NA11OAR4320072, U.S. Department of Commerce. The statements, findings, conclusions, and recommendations are those of the authors and do not necessarily reflect the views of NOAA or the U.S. Department of Commerce. prior to any data collection exploiting electronic beam steering. The antenna beamwidth is $1.5^{\circ}$ at boresite and gradually increases to $2.1^{\circ}$ at $\pm 45^{\circ}$ from boresite. The peak transmitted power is $750 \mathrm{~kW}$ and range resolution cells are produced with a 240-m spacing. In some aspects, such as beamwidth and sensitivity, the NWRT PAR is inferior compared to operational radars such as the Weather Surveillance Radar-1988 Doppler (WSR-88D). However, the purpose of this system is not to achieve operational-like performance or to serve as a prototype for the replacement of WSR-88D radars, but to demonstrate the operational utility of some of the unique capabilities offered by PAR technology that may eventually drive the design of future operational weather radars [2].

The deployment of new signal processing hardware on the NWRT PAR [3] marked the beginning of a series of engineering upgrades. Since then, significant hardware, software infrastructure, and signal processing upgrades have been completed to support the system's mission as a demonstrator for the MPAR concept. Using a path of continuous development with an average of two software releases every year, new and improved capabilities have been made available on the NWRT PAR in recent years [4-7]. The need for these improvements is twofold. On one hand, it is desirable that the NWRT PAR produces data with quality as close as possible to that of the WSR-88D. High data quality leads to better data interpretation and is conducive to the development of more effective automatic algorithms. On the other hand, software infrastructure and signal processing improvements are needed to demonstrate new capabilities, some of which that are applicable to both conventional and phased-array radars, and some that are unique or better suited to PARs. A prime example of the latter is the use of adaptive scanning strategies to perform focused and tailored observations of the atmosphere. Whereas adaptive scanning is not unique to PAR, update times can be greatly reduced by using PAR's electronic beam steering capabilities because scanning strategies are not constrained by the inherent mechanical inertia of reflector antennas.

\section{ADAPTIVE SCANNING}

Adaptive weather scanning consists of changing the radar's scanning strategy in response to changes in the atmosphere and can be exploited to improve the value of weather radar observations used by national-weather-service (NWS) forecasters. Radar data can be characterized by their temporal and spatial sampling and their quality. In principle, update 
time, spatial sampling (i.e., the number of beam positions to scan), and data quality (e.g., variance of meteorologicalvariable estimates, absence of artifacts and sampling ambiguities) are mutually coupled criteria in the design of scanning strategies for weather radars. That is, if one is to be improved, one or both of the others must be sacrificed [8]. However, through adaptive scanning, the best tradeoffs can be dynamically realized for each particular situation.

The concept of adaptive scanning can be applied to both reflector and phased-array antenna systems; however, a PAR using electronic beam steering is better suited for it since it is not constrained by mechanical inertia. The current implementation of adaptive scanning on the NWRT comprises three functions: focused observations, tailored observations, and scheduling. Focused observations provide a means to reduce update times with no sacrifice in data quality or spatial coverage by devoting less radar time to regions of reduced interest (e.g., clear air). Tailored observations ensure that the radar resources (i.e., time) spent observing a specific storm or cluster of storms are effectively used to provide the best data possible with the purposes of supporting the forecaster's warning-decision process. Finally, a scheduler is required to interlace different types of observations in time in order to provide the desired update times. In summary, adaptive scanning can be exploited to optimize the sampling and quality of radar data as a function of the weather scenario and the impact of different radar-data characteristics on the forecaster's warning-decision process.

\section{A. Focused Observations}

Scientists at the NSSL have been exploring the hightemporal resolution weather scanning capabilities of the agilebeam NWRT PAR for a number of years. Fast adaptive scanning with the NWRT PAR was first demonstrated in 2009 with the development and real-time implementation of the Adaptive DSP Algorithm for Phased-Array Radar Timely Scans (ADAPTS). Detecting regions with significant weather returns is the key for adaptive focused observations; and ADAPTS works in real time by classifying individual beam positions within a scanning strategy as active or inactive based on two significance criteria: intrinsic and neighborhood. A beam position that exhibits reflectivity values above a (predefined, range-dependent) threshold while covering a largeenough region of space is said to have intrinsic significance. A

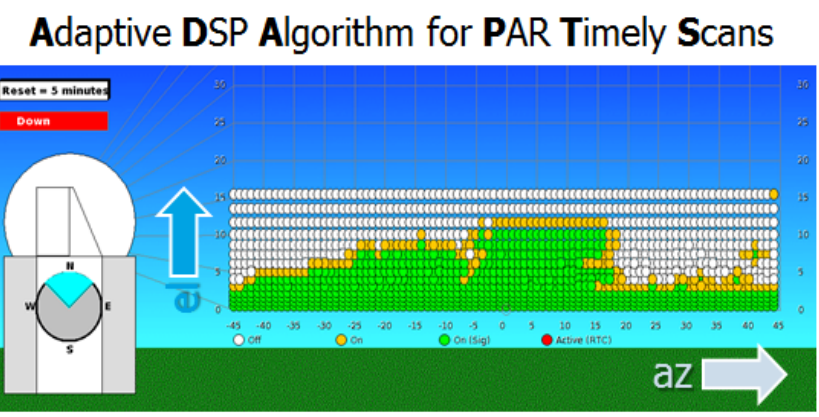

Real-time display of active /inactive beam positions

$00 \quad 0$

Fig. 1. Example of active/inactive classification of beam positions with ADAPTS through the NWRT PAR user interface. beam position in the vicinity of another beam position with intrinsic significance is said to have neighborhood significance. Thus, active beam positions are those in which significance either has already been established (intrinsic) or is expected to be established in the next revisit due to storm growth and advection from intrinsically significant neighboring beam positions. Fig. 1 shows an example of active and inactive beam positions as determined by ADAPTS where green dots represent intrinsically active beam positions, orange dots represent active beam positions based on neighborhood, and white dots represent inactive beam positions.

ADAPTS uses its classification of beam positions into active or inactive to redefine the scanning strategy that the radar will use next. In the ADAPTS scanning strategy, active beam positions are devoted the most radar time, whereas the opposite is true for inactive beam positions. Inactive beam positions should receive the minimum amount of radar time but must be periodically revisited in order to maintain the radar's ability to detect new developments. In its current implementation, ADAPTS completely removes inactive beam positions from the scanning strategy but the timeline includes a separate weather detection scan that is scheduled to run periodically but at a slower rate than the weather surveillance scan.

Revisiting only active beam positions leads to the fastest update times, but the time savings afforded by ADAPTS depends on the distribution of weather echoes in the scan volume. For example, isolated storms at far distances from the radar lead to the greatest time savings, while widespread precipitation, squall lines almost perpendicular to the radar beam, or storms close to the radar are examples where ADAPTS may not reduce scan times significantly.

\section{B. Tailored Observations}

Adapting the characteristics of the scanning strategy to match the characteristics of the weather phenomena being observed is a complex problem and one of the goals of the Phased-Array Radar Innovative Sensing Experiments (PARISE), which are conducted every spring at the NSSL with forecasters from the National Weather Service [9]. In the current implementation of adaptive scanning on the NWRT PAR different sub-volumes are sampled using different scanning strategies depending on whether they contain clearair, non-severe precipitation, or severe precipitation echoes. Whereas ADAPTS already provides a means to identify clear air from precipitation echoes, it does not differentiate between stratiform (non-severe) and convective (severe) regions. Thus, a convective-storm cluster identification algorithm was developed for this purpose [10]. An example of its performance is provided in Fig. 2.

While a comprehensive set of rules to tailor the temporal and spatial sampling and the quality of the data collected for specific storm types have not been explicitly defined, a natural way to tailor the scanning strategy for the most severe storms is to provide faster updates. This agrees with the typical needs in severe weather situations, where forecasters focus their attention on the most intense (convective) storms. The current implementation of adaptive scanning can be summarized in 


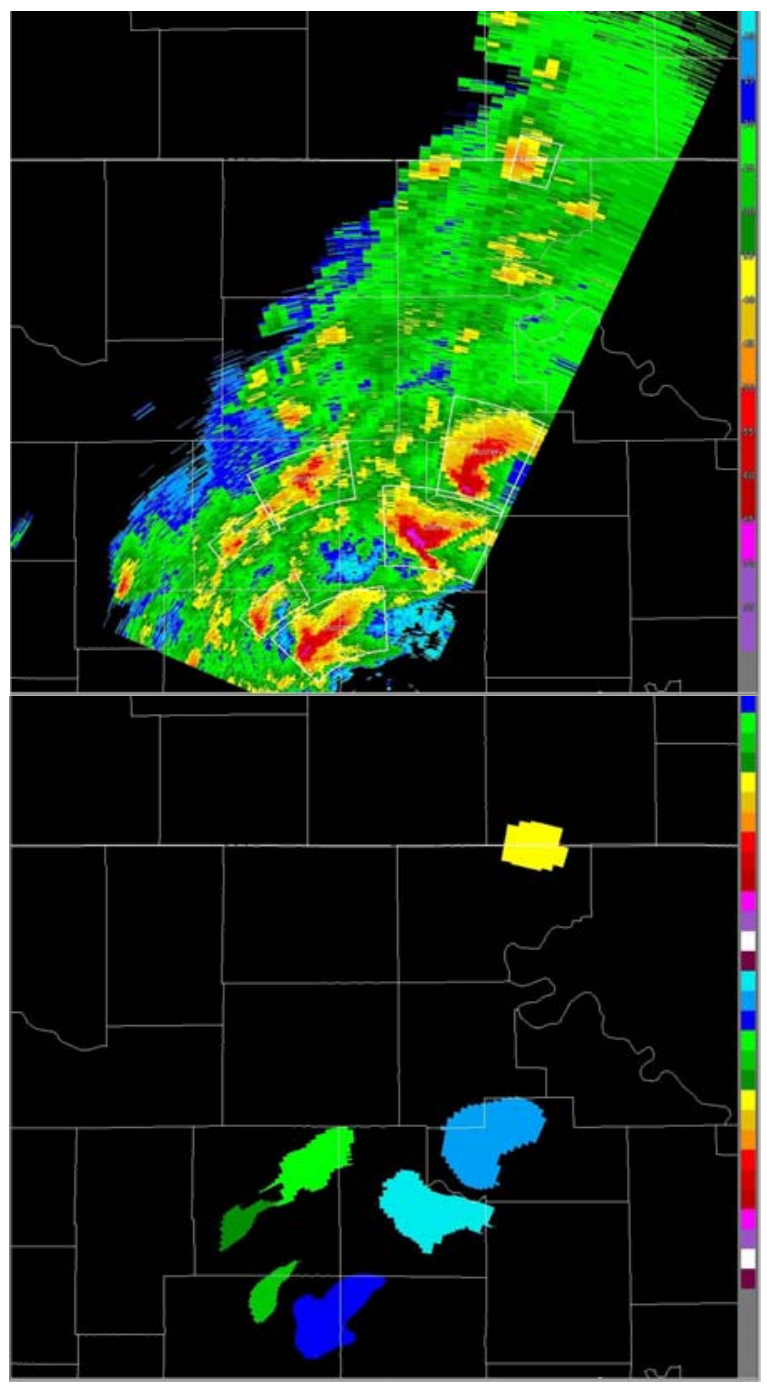

Fig. 2. Example of the convective-storm cluster identification algorithm output (bottom) using reflectivity field as input (top).

Table I. Future implementations of adaptive scanning will address more complex tailoring mechanisms, including changes to the vertical sampling and changes to the pulse repetition time (PRT) as a function of the range of storms.

\section{Scheduling}

Having defined the scanning strategy characteristics for clear-air, non-severe weather and severe weather, it is now important to describe how these are scheduled to optimize radar resources and produce the desired update times. In the current implementation of adaptive scanning, only one severe storm (or cluster of storms) can be selected for faster update times. Thus, radar operators use the output of the storm-cluster identification algorithm (similar to the bottom panel in Fig. 2) to select the convective cluster that will be assigned more radar time. The resulting timeline is illustrated in Fig. 3 and described next.
TABLE I. CURRENT ADAPTIVE SCANNING CAPABILITIES OF THE NWRT PAR

\begin{tabular}{|l|c|c|c|}
\hline \multirow{2}{*}{$\begin{array}{c}\text { Scanning } \\
\begin{array}{c}\text { Strategy } \\
\text { Characteristic }\end{array}\end{array}$} & Clear Air & $\begin{array}{c}\text { Non-Severe } \\
\text { Weather }\end{array}$ & $\begin{array}{c}\text { Severe } \\
\text { Weather }\end{array}$ \\
\cline { 2 - 4 } Update time & Slow & Normal & Fast \\
\hline Spatial sampling & Coarse & Normal & Normal \\
\hline Data quality & Poor & Good & Good \\
\hline
\end{tabular}

After the radar operator chooses the preferred spatial sampling (beam positions defined by their azimuth and elevation coordinates) and corresponding acquisition parameters (pulse repetition time(s) and number of samples), the radar runs a scanning strategy based on these characteristics where all beam positions are active (denoted by FULL in Fig. 3 ). This strategy is used to classify sub-volumes as containing clear-air or weather echoes. ADAPTS is used on subsequent runs to focus observations on sub-volumes with weather echoes with typical update times of $\sim 60 \mathrm{~s}$. Data from this strategy are used to further classify weather into non-severe and severe categories. Depending on the extent of weather echoes (i.e., the footprint of active beam positions) the radar may have time to run one or more scanning strategies that focus on the severe storm (or cluster of storms) selected by the user with typical scan times of $\sim 10 \mathrm{~s}$ (CLUSTER in Fig. 3). About every $150 \mathrm{~s}$, a separate detection scan (DET in Fig. 3) is scheduled to catch any new developments in the areas covered by (up until that point) inactive beam positions. This detection scan is very quick $(\sim 7 \mathrm{~s})$, but the data it produces are only used for detection purposes and not sent to displays or other algorithms. Except for the full scan at the beginning, this basic timeline repeats indefinitely while adapting to changes in the atmosphere. That is, the ADAPTS scan is continually modified by two mechanisms. First, it responds to storm evolution via the neighborhood significance criterion, and second, it responds to storm initiation via the information produced by the detection scan. Also, the CLUSTER scan is redefined every time an ADAPTS scan runs. A full scan is required only after the spatial sampling is changed by the radar operator or until the antenna pedestal moves. In future implementations, more advanced scheduling algorithms will be used [e.g., 11] so that multiple severe storms can be sampled with independent update times concurrently.

As mentioned before, the NWRT PAR is limited to scanning a $90^{\circ}$ azimuthal sector at any given moment. Thus, an adaptive pedestal control algorithm was developed to keep track of the relative position of the storm(s) of interest in the $90^{\circ}$ sector [12]. When necessary, this algorithm commands the pedestal to a new position to maintain coverage of the convective storm (or storm cluster) of interest. The timeline described above is restarted upon a change in the pedestal position and the ADAPTS database of active/inactive beam positions is reinitialized to all active beams.

Animations using real data cases sampled with the latest adaptive scanning capabilities of the NWRT PAR will be shown at the conference. 


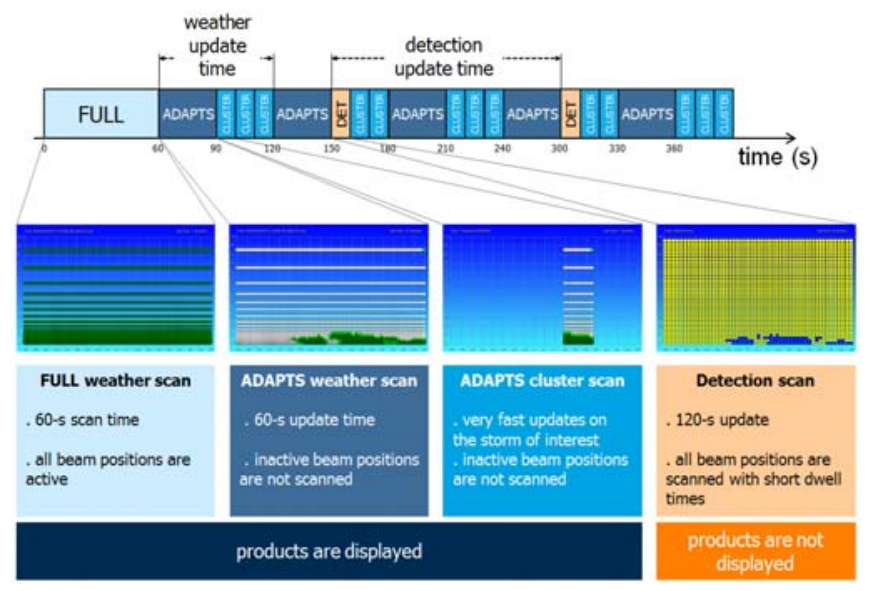

Fig. 3. Example of the adaptive scanning timeline the combines focused observations (ADAPTS) and tailored observations (Table I).

\section{SUMMARY AND FUtURE WORK}

Weather radar adaptive scanning has the potential to reduce revisit times and to provide meteorological data that can aid in forecaster's warning-decision processes. The current implementation of adaptive scanning on the NWRT PAR exploits electronic beam steering to perform focused and tailored observation of the weather. A simple scheduler is used to provide the desired update times for each sub-volume time and to maintain surveillance of the inactive areas in order to detect storm initiation. It has been demonstrated that a phasedarray weather radar can produce higher temporal resolution data without sacrificing data quality or spatial resolution through more efficient use of radar resources. This is one step further towards demonstrating that PAR technology can be exploited to achieve performance levels that are not feasible with current weather-radar operational technology. Nonetheless, more research is needed to translate these improvements into concrete, measurable, and meaningful service improvements for the National Weather Service and other government agencies. As such, the NWRT PAR will continue to explore and demonstrate new capabilities to address $21^{\text {st }}$ century weather-forecast and warning needs.

\section{REFERENCES}

[1] M. Weber, J. Cho, J. Herd, J. Flavin, W. Benner, and G. Torok, "The Next-Generation Multimission U.S. Surveillance Radar Network," Bull. Amer. Meteor. Soc., vol. 88, pp. 1739-1751, 2007.
[2] D. S. Zrnić, J. F. Kimpel, D. E. Forsyth, A. Shapiro, G. Crain, R. Ferek, J. Heimmer, W. Benner, T. J. McNellis, R. J. Vogt, "Agile beam phased array radar for weather observations," Bull. Amer. Meteor. Soc., vol. 88, pp. 1753-1766, 2007.

[3] D. E. Forsyth, J. F. Kimpel, D. S. Zrnić, R. Ferek, J. F. Heimmer, T. McNellis, J. E. Crain, A. M. Shapiro, R. J. Vogt, and W. Benner, "Update on the National Weather Radar Testbed (Phased-Array)," in Proc. 33rd Conf. Radar Meteorology, Cairns, Australia, 2007, paper 7.2.

[4] S. Torres, C. Curtis, I. Ivić, D. Warde, E. Forren, J. Thompson, D. Priegnitz, and R. Adams, "Update on signal processing upgrades for the National Weather Radar Testbed," in Preprints 25th Int. Conf. Interactive Information and Processing Systems (IIPS) for Meteor., Oceanography, and Hydrology, Phoenix, AZ, 2009, paper 8B.4.

[5] S. Torres, C. Curtis, I. Ivić, D. Warde, E. Forren, J. Thompson, D. Priegnitz, and R. Adams, "Update on signal processing upgrades for the National Weather Radar Testbed," in Preprints 26th Int. Conf. Interactive Information and Processing Systems (IIPS) for Meteor., Oceanography, and Hydrology, Atlanta, GA, 2010, paper 14B.2.

[6] S. Torres, C. Curtis, I. Ivić, D. Warde, E. Forren, J. Thompson, D. Priegnitz, and R. Adams, "Software and signal processing upgrades for the National Weather Radar Testbed phased-array radar," in Preprints 27th Conf. Interactive Information and Processing Systems (IIPS) for Meteorology, Oceanography, and Hydrology, Seattle, WA, 2011, paper 12B.3.

[7] S. Torres, P. Heinselman, R. Adams, C. Curtis, E. Forren, I. Ivić, D. Priegnitz, J. Thompson, and D. Warde, "ADAPTS Implementation: Can we exploit phased-array radar's electronic beam steering capabilities to reduce update times?," in Preprints 28th Conf. Interactive Information and Processing Systems (IIPS) for Meteorology, Oceanography, and Hydrology, New Orleans, LA, 2012, paper 6B.3.

[8] P. Heinselman, and S. Torres, "High-temporal resolution capabilities of the National Weather Radar Testbed phased-array radar," J. Applied Meteor., vol. 50, pp. 579-593, 2011.

[9] P. Heinselman, D. LaDue, D. M. Kingfield, R. Hoffman, and B. W. MacAloney II, "Simulated NWS tornado warning decisions using rapidscan radar data," in Preprints 29th Conf. Environmental Information Processing Technologies, Austin, TX, 2013, paper 8.3.

[10] D. Priegnitz, S. Torres, and P. Heinselman, "Enhancements to the National Weather Radar Testbed Phased Array Radar storm tracking function," in Preprints 29th Conf. Environmental Information Processing Technologies, Austin, TX, 2013, paper 19.

[11] R. Reinoso-Rondinel, T.-Y. Yu, and S. Torres, "Multifunction phasedarray radar: time balance scheduler for adaptive weather sensing," $J$. Atmos. Oceanic Technol., vol. 27, pp. 1854-1867, 2010.

[12] D. Priegnitz, S. Torres, and P. Heinselman, "An adaptive pedestal control algorithm for the National Weather Radar Testbed Phased Array Radar," in Preprints 28th Conf. Interactive Information and Processing Systems (IIPS) for Meteorology, Oceanography, and Hydrology, New Orleans, LA, 2012, paper P1.7. 